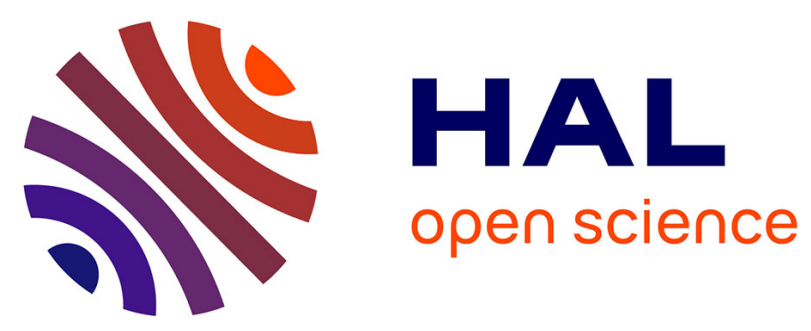

\title{
Iuliacumite: A Novel Chemical Short-Range Order in a Two-Dimensional Wurtzite Single Monolayer InAs 1- x Sb x Shell on InAs Nanowires
}

M. Schnedler, T. Xu, Isabelle Lefebvre, J.-P. Nys, S R Plissard, Maxime Berthe, H. Eisele, R. E Dunin-Borkowski, Ph. Ebert, B. Grandidier

\section{To cite this version:}

M. Schnedler, T. Xu, Isabelle Lefebvre, J.-P. Nys, S R Plissard, et al.. Iuliacumite: A Novel Chemical Short-Range Order in a Two-Dimensional Wurtzite Single Monolayer InAs 1- x Sb x Shell on InAs Nanowires. Nano Letters, 2019, 19 (12), pp.8801-8805. 10.1021/acs.nanolett.9b03584 . hal-03036942

\section{HAL Id: hal-03036942 https://hal.science/hal-03036942}

Submitted on 2 Dec 2020

HAL is a multi-disciplinary open access archive for the deposit and dissemination of scientific research documents, whether they are published or not. The documents may come from teaching and research institutions in France or abroad, or from public or private research centers.
L'archive ouverte pluridisciplinaire HAL, est destinée au dépôt et à la diffusion de documents scientifiques de niveau recherche, publiés ou non, émanant des établissements d'enseignement et de recherche français ou étrangers, des laboratoires publics ou privés. 


\title{
luliacumite: a novel chemical short range order
}

\section{in a two-dimensional wurtzite single monolayer $\operatorname{InAs} s_{1-x} S_{b_{x}}$ shell on InAs nanowires}

\author{
M. Schnedler, ${ }^{*, \dagger}$ T. Xu, ${ }^{\ddagger}$ I. Lefebvre,,$^{\ddagger}$ J.-P. Nys, ${ }^{\ddagger}$ S. R. Plissard, ${ }^{\ddagger}$ M. Berthe, ${ }^{\ddagger} H$.
} Eisele, ${ }^{\|}$R. E. Dunin-Borkowski, ${ }^{\dagger}$ Ph. Ebert, ${ }^{\dagger}$ and B. Grandidier, ${ }^{*}$,

†Peter Grünberg Institut, Forschungszentrum Jülich GmbH, 52425 Jülich, Germany $\ddagger$ Université Lille, CNRS, Centrale Lille, ISEN, Université Valenciennes, UMR 8520 IEMN, F-59000 Lille, France

\Key Laboratory of Advanced Display and System Application, Shanghai University, 149 Yanchang Road, Shanghai 200072, People's Republic of China $\S C N R S$-Laboratoire d'Analyse et d'Architecture des Systèmes (LAAS), Université de Toulouse, 7 Avenue du Colonel Roche, 31400 Toulouse, France || Institut für Festkörperphysik, Technische Universität Berlin, Hardenbergstr. 36, 10623 Berlin E-mail: m.schnedler@fz-juelich.de; bruno.grandidier@isen.iemn.univ-lille1.fr

\begin{abstract}
A chemical short range order is found in single monolayer $\operatorname{InAs}_{1-\mathrm{x}} \mathrm{Sb}_{\mathrm{x}}$ shells, which inherit a wurtzite structure from the underlying InAs nanowire, instead of crystallizing in the energetically preferred zincblende structure. The chemical order is characterized by an anti-correlation ordering vector in $\langle 11 \overline{2} 0\rangle$ direction and arises from strong Sb-Sb repulsive interactions along the atomic chains in $\langle 11 \overline{2} 0\rangle$ direction.
\end{abstract}




\section{Keywords}

Chemical ordering; Single monolayer; Wurtzite In(As,Sb); Nanowire shell

The reduced dimensionality of semiconductor nanowires (NWs) offers the unique opportunity to grow materials with crystal structures, which are otherwise unstable. One of the most prominent example is the growth of wurtzite (WZ) structure InAs NWs, 1 although stable InAs bulk material prefers the zincblende (ZB) structure. In contrast, other III-V materials, notably $\mathrm{InAs}_{1-\mathrm{x}} \mathrm{Sb}_{\mathrm{x}}$, prefer to keep their ZB bulk structure even in NWs or nanostructures with reduced dimensionality. ${ }^{213}$ However, lateral overgrowth of these ternary III-V semiconductor alloys on sidewall facets of WZ structure III-V nanowires offers the prospect to nevertheless obtain reliably a WZ structure shell, despite being unstable. Thereby new polytype structures of ternary III-V semiconductor alloys can be achieved, offering additional degrees of freedom for adjusting the band structure in, e.g., core-shell nanowires.

In addition of controlling the polytype structure, controlling ordering of the different chemical species in ternary III-V semiconductor alloys offers further means to tune the electrical properties. This has been amply demonstrated for ZB structure III-V semiconductors, where a variety of different chemical ordering structures were identified (i.e. chalcopyrite, $\mathrm{CuPt}, \mathrm{CuAu}$, famatinite, triple period, and lazarevicite). ${ }^{[4} 9$ In contrast, rather little is known about chemical ordering in WZ structure III-V semiconductors. Thus far most reports on chemical ordering refer to $c$ axis ordering and superlattices in WZ structure group III-nitride semiconductors. ${ }^{10}$-17 Only during growth on pyramidal pits ordering along the pit's semipolar growth facets was observed. $\frac{18}{1 n}$ In contrast to the limited experimentally observed ordering structures in WZ materials, theory predicts a large variety of possible ordered WZ structures, with ordering vectors not only in $c$ direction ${ }^{19120}$, but without experimental verification yet.

Furthermore, thus far chemical ordering in III-V semiconductor alloys was experimentally identified in (quasi) three-dimensional materials, i.e. bulk or extended nanowire bulk-like sections, However, lateral overgrowth on NW sidewall facets offers the possibility to ob- 
tain monolayer thin two-dimensional ternary III-V semiconductor sheets. At present it is unclear if any chemical ordering is forming in such two-dimensional WZ-structure III-V semiconductors or in general in emerging group of two-dimensional ternary semiconductor materials. $21+23$

Therefore, we designed a two-dimensional single monolayer InAs $\mathrm{s}_{1-\mathrm{x}} \mathrm{Sb}_{\mathrm{x}} \mathrm{WZ}$ structure shell on sidewall facets of InAs nanowires and investigated its chemical ordering using atomically resolved scanning tunneling microscopy (STM). We identify the existence of a short-range chemical ordering in this WZ structure single monolayer shell. The new type of ordering in a two-dimensional sheet, called Iuliacumite, is characterized by an anti-ordering vector in $\langle 11 \overline{2} 0\rangle$ direction. The ordering is driven by a strong repulsive interaction of neighboring Sb atoms along the $\langle 11 \overline{2} 0\rangle$-oriented atomic chains on the $m$ plane sidewall facets.

In order to fabricate two-dimensional single WZ structure III-V semiconductor monolayers, [0001] oriented WZ InAs NW segments, were overgrown in-situ by $\operatorname{InAs}_{1-x} \mathrm{Sb}_{x}$ at $410^{\circ} \mathrm{C}$ using gas source molecular beam epitaxy. After growth, a thin capping layer of As was deposited onto the nanowires' surfaces to prevent oxidation during transport through air into the analysis system. There, the nanowires were heated under UHV conditions to remove the capping layer. After cleaning, the NWs were cleaved-off and transferred to a freshly cleaned $\mathrm{Si}(111)$ surface, to investigate their sidewalls in-situ by STM. 219.

Figure1(a) illustrates schematically the structure of the investigated NWs, with the WZ structure InAs core in blue and the axially grown $\operatorname{InAs}_{1-x} \mathrm{Sb}_{x} \mathrm{ZB}$ structure segment in red. As illustrated below, during axial growth also a lateral overgrowth of WZ structure $\mathrm{InAs}_{1-x} \mathrm{Sb}_{x}$ on the InAs NW sidewall facets occurs (light blue). Here we focus on these overgrown (101̄0) sidewall facets. Figure1(b) illustrates an atomically resolved filled-state STM image of a sidewall facet acquired at negative sample bias. Under these conditions, the filled dangling bonds above every group $\mathrm{V}$ atom are imaged. First of all, the symmetry and lattice constants demonstrate that the overgrown layer kept the WZ structure of the InAs core. ${ }^{24}$ Second, the surface exhibits a high density of atomically sized protrusions (see 

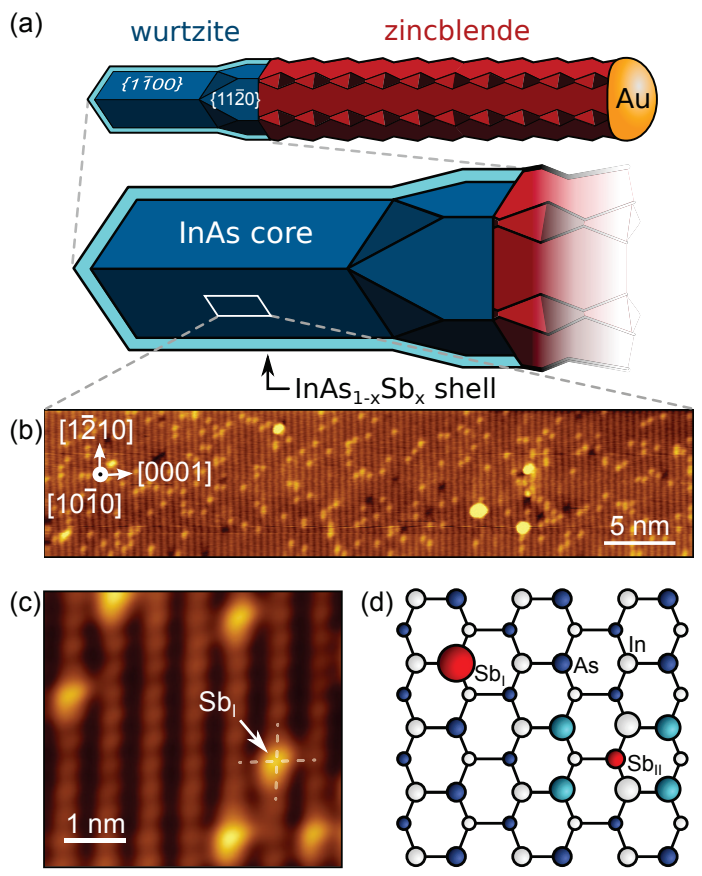

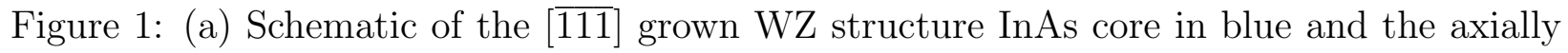
grown InAs $\mathrm{As}_{1-x} \mathrm{Sb}_{x} \mathrm{ZB}$ structure segment in red. During axial growth an additional lateral overgrowth on the sidewall facets of the InAs core by WZ structure InAs $\mathrm{s}_{1-x} \mathrm{Sb}_{x}$ occurs (light blue). (b) Atomically resolved constant-current STM image of the overgrown sidewall surface measured at $77 \mathrm{~K}$, using a sample voltage of $-3 \mathrm{~V}$ and a tunnel current of $10 \mathrm{pA}$. Under these tunneling conditions, the filled dangling bond states above the surface anions are imaged. (c) Magnified STM image showing the atomic rows in detail. The bright atomically localized contrast features arise from substitutional Sb atoms on As sites. (d) Atomic ball model of $\mathrm{Sb}$ atoms in different subsurface layers (same orientation as the STM image), illustrating different symmetries of the Sb contrast on the surface: Sb atoms incorporated on As sites in the surface layer (denoted $\mathrm{as} \mathrm{Sb}_{\mathrm{I}}$ ) can be observed as protrusions centered on top of an anion sublattice site in the STM image. In contrast, Sb atoms incorporated in the second atomic layer $\left(\mathrm{Sb}_{\mathrm{II}}\right)$ can be anticipated to give rise to an outward relaxation of four surface As atoms (light blue balls) and thus a protrusion centered inbetween of the atomic chains. Since such contrast features are never observed, the second atomic layer is free of $\mathrm{Sb}$, indicating the presence of only a single monolayer thick $\operatorname{InAs}_{1-x} \mathrm{Sb}_{x}$ shell. 
magnification in Fig. 1(c)), arising from the presence of substitutional Sb atoms on As lattice positions. The contrast arises primarily from the outward relaxation induced by the Sb atoms with larger atomic radii as compared to the As atoms of the host lattice, in analogy to Sb atoms in ZB structure (110) III-V surfaces. 25

At closer look, all protrusions exhibit identical symmetries and intensities, centered exactly on top of a surface As sublattice position. Considering the crystal symmetry, substitutional $\mathrm{Sb}$ atoms in different layers in and below the surface exhibit different symmetries of the Sb contrast on the surface (Fig.1(d)). Protrusions centered on top of surface As sublattice sites are compatible with Sb atoms incorporated on As sites in the surface layer, as schematically illustrated in the atomic model for the case labeled $\mathrm{Sb}_{\mathrm{I}}$ in Fig.11(d). In contrast, a $\mathrm{Sb}$ atom in the second layer $\left(\mathrm{Sb}_{\mathrm{II}}\right)$ is centered in between of four surface $\mathrm{As}$ atoms, which can be anticipated to relax outward due to the larger Sb atom (as indicated by the larger size of the slightly lighter blue balls representing the neighboring As atoms in Fig.1(d)), in analogy to impurities in ZB structure (110) III-V semiconductor surfaces. $\underline{26}+29$ Hence, a $\mathrm{Sb}$ atom in the second layer would create a bright contrast with a mirror symmetry in between of the atomic rows along the $c$ direction. Such a contrast is never observed, even not in local areas free of surface Sb atoms. Hence, Sb is not present in the second layer, indicating that the laterally overgrown $\operatorname{InAs}_{1-x} \mathrm{Sb}_{x}$ shell is a two-dimensional sheet, only a single monolayer thick. A count of the Sb protrusions yield concentrations ranging between $6.3-9.8 \%$ for our NWs.

This raises the question why the sidewall overgrowth by WZ structure $\operatorname{InAs}_{1-x} \mathrm{Sb}_{x}$ is limited to only one monolayer at our growth conditions. This limitation implies that nuclei of islands on top of the single monolayer shell are unstable and hence Sb atoms prefer incorporation in the axially grown upper ZB structure $\mathrm{InAs}_{1-x} \mathrm{Sb}_{x}$ section. This is conceivable, since $\mathrm{InAs}_{1-x} \mathrm{Sb}_{x}$ prefers the $\mathrm{ZB}$ structure even at very small Sb concentrations. However, overgrowth on sidewalls facets of WZ structure InAs NWs forces $\mathrm{InAs}_{1-x} \mathrm{Sb}_{x}$ to crystallize in the WZ structure of the InAs core. In addition, an inward diffusion of Sb is energetically 
prohibited $^{30}$ and $\mathrm{Sb}$ as surfactant has the tendency to float, 31 both leading to incorporation only in the surface layer and hence stabilizing the single monolayer $\operatorname{InAs}_{1-x} \mathrm{Sb}_{x} \mathrm{WZ}$ structure shell.

The above discovery of a single monolayer shell raises the question, whether (short range) chemical ordering can emerge and how it might look like in such two-dimensional WZ structure InAs $\mathrm{s}_{1-x} \mathrm{Sb}_{x}$ monolayer sheets. For identifying a possible short range order, we mapped the spatial distribution of the group $\mathrm{V}$ elements in the $\mathrm{InAs}_{1-x} \mathrm{Sb}_{x}$ monolayer shell with atomic resolution. This chemical map is then used for an in-depth analysis of the spatial correlation of the Sb distribution. Figure 2(a) illustrates the two-dimensional pair-correlation function $(\mathrm{PCF}) c(x, y)$ for $\mathrm{Sb}$ atoms. Values close to one represent statistically expected $\mathrm{Sb}$ pair distributions without interactions. Hence the data shows that at larger distances no interactions are present. However, for nearest neighbor Sb pairs along the $a$ direction the PCF exhibits values much smaller than 1, indicating a low probability of occurrence (for quantitative comparability see Fig.2(b)). In contrast, nearest neighbor Sb pairs in $c$ direction, i.e. across the atomic chains, exhibit PCF values slightly larger than 1, indicating slightly more frequent occurrences than statistically expected. The results emphasize that a strong anti-correlation in [12 10$]$ and only a weak correlation in [0001] direction define the chemical short range order. This yields an anti-ordering vector $\underline{v}_{\mathrm{AO}}=\frac{2}{3} a[1 \overline{2} 10]$ and an ordering vector $\underline{v}_{\mathrm{O}}=c[0001]$.

In order to investigate possible Sb concentration effects, we extracted the pair correlation values for nearest neighbor Sb pairs in a direction for local surface areas with low (6.3\%) and with higher (9.8\%) Sb concentration. We obtained values of 0.512 and 0.528 for $6.3 \%$ and 9.8\% Sb, respectively. Hence, this indicates that the anti-correlation ordering in $a$ direction is concentration independent.

For a further understanding of the underlying physical mechanisms driving the ordering, we derive the so-called mean force potential $W(x, y)=-k T \ln [c(x, y)]^{32}$, which is a first approximation of the pair interaction potential in the low density limit. $k$ is the Boltzmann 

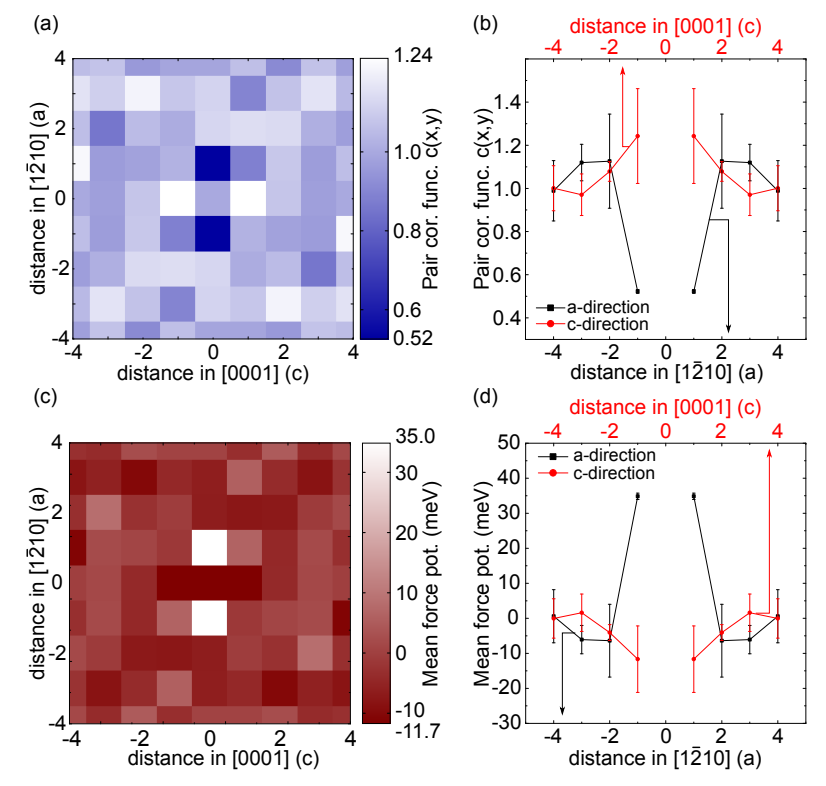

Figure 2: (a) Heatmap of the two-dimensional Sb pair correlation function $c(x, y)$ within the WZ structure InAs $\mathrm{s}_{1-x} \mathrm{Sb}_{x}$ single monolayer sheet. Values above (below) 1 indicate a higher (lower) than statistically expected occurrence of Sb pairs without interaction (scale on the right). (b) Cross sections of the $\mathrm{PCF} c(x, y)$ along ( $a$ direction) and across (c direction) the atomic rows. For nearest neighbor $\mathrm{Sb}$ pairs along the $a$ direction the PCF exhibits values much smaller than 1, indicating a strong repulsion. (c) Heatmap of the two-dimensional mean force potential $W(x, y)=-k T \ln [c(x, y)]$ derived from the experimentally measured PCF. (d) Cross sections of the mean force potential in $a$ and $c$ directions. Nearest neighbor $\mathrm{Sb}$ pairs in $a$ direction exhibit a repulsive interaction energy of about $+35 \mathrm{meV}$, whereas in $c$ direction an weak attractive interaction energy of about $-12 \mathrm{meV}$ occurs. 
constant and $T$ the freeze-in temperature, below which no diffusion or site changes within the $\mathrm{InAs}_{1-x} \mathrm{Sb}_{x}$ monolayer occur anymore. An upper limit for $T$ is assumed to be $623 \mathrm{~K}$, the temperature used for evaporation of the As cap. The resulting mean force potential, shown in Figs.2(c) and (d), reveal a strong Sb-Sb repulsion with a repulsive energy of about $35 \mathrm{meV}$ in $a$ direction and a Sb-Sb attraction with an attractive energy of about $12 \mathrm{meV}$ in $c$ direction. Hence, the energy difference between both configurations is approximately $(47 \pm 11) \mathrm{meV}$.

This value is compared to density function theory computations of the total energy difference of an $\operatorname{InAs}(10 \overline{1} 0)$ surface layer that exhibits nearest neighbor Sb-Sb pairs in either $c$ or $a$ direction. The calculations were carried out using the Vienna ab-initio simulation program (version 5.2.11). $\stackrel{33}{ }$ All electron projector augmented wave (PAW) pseudopotentials were taken ${ }^{34}$ using the Ceperley and Alder approximation,, 35 parameterized by Perdew and Zunger for exchange and correlation energies (i.e. the Sb, As, In, H1.25 and H.75 PAW potentials).9 We used a kinetic energy cutoff of $312.5 \mathrm{eV}$. The calculations were performed for $(10 \overline{1} 0)$ oriented supercells with different sizes of $4 \times 4,4 \times 5$, and $5 \times 5$ and thicknesses of 8 as well as 10 monolayers ( $\mathrm{H}$ terminated on back side). The supercells were optimized until forces on atoms were smaller than $0.9 \mathrm{meV} / \AA$. The calculations yield similar total energies for all supercells. The average energy difference between $\mathrm{Sb}-\mathrm{Sb}$ nearest neighbors in $c$ and $a$ direction is found to be $24 \mathrm{meV}$, with pairs in $c$ direction being lower in energy. Note, nearest neighbor Sb-Sb pairs along the zigzag chains on the cubic ZB (110) surfaces exhibit a very similar bonding structure and indeed a very similar interaction energy of $33 \mathrm{meV}$ was calculated and experimentally verified..$^{[}$

The calculated and experimental energy differences for pairs in $c$ and $a$ directions are in the same order, especially the energy for pairs in $c$ direction is lower than for those in $a$ direction. This can be understood by considering the the incorporation of Sb atoms with larger atomic radii in the WZ crystal structure: In $c$ direction the bonding structure is less rigid and nearest neighbor $\mathrm{Sb}$ atoms on the surface are in fact second nearest neighbor group 
V atoms (neglecting the interbonded group III atoms), since only every second (0001) plane is visible in filled (or empty) state STM images (see schematic in Fig.1(d)). In contrast, for $\mathrm{Sb}$ pairs along the $a$ direction the $\mathrm{Sb}$ atoms are much closer since they are nearest neighbor group V atoms separated only by one group III atom. This enhances strain for such $\mathrm{Sb}$ pairs, in analogy with similarly bonded Sb pairs along the zigzag chain on ZB structure (110) surfaces. 9

At this stage we compare quantitatively the measured and calculated energy differences between the Sb pairs in $c$ and $a$ direction. The experimental value is somewhat larger than the calculated one. However, the experimental determination suffers from the uncertainty of the freeze-in temperature and from many body effect in the pair interactions. First, the freeze-in temperature is an upper limit and thus lower in reality. Hence, the measured energy difference is likely overestimated and can be anticipated to be lower by roughly 10 to $15 \mathrm{meV}$. Second, at the given Sb concentrations and interaction ranges, many body effects in $\mathrm{Sb}-\mathrm{Sb}$ interaction can be anticipated to be present, since the ratio between concentration and interaction length is in analogy to that of dopant atoms in GaAs, where many body effects were shown to affect pair correlation functions. $\frac{\sqrt{36}}{}$ Hence, the mean field potential cannot be assumed to be equal to the pair interaction energy anymore and thus many body effects affect the measured energy difference. Although a quantitative agreement of the measured and calculated pair interaction energies is difficult to reach due to the mentioned effects, both, the experimental and theoretical results lead to the same physical conclusion, i.e. the presence of a novel short range order in a two-dimensional WZ structure $\operatorname{InAs}_{1-x} \mathrm{Sb}_{x}$ monolayer shell.

The experimental results yielded a very pronounced anti-correlation short-range ordering along the $a$ direction which is found to be independent of the Sb concentration. On this basis, we derive an idealized long-range chemical ordering structure of the monolayer shell on the InAs sidewall facet, reached at the compositional limit of $\operatorname{InAs}_{0.5} \mathrm{Sb}_{0.5}$ in the top plane (lower planes are pure InAs). This idealized ordering structure is given schematically in side and 
top view in Fig. 3(a) and (b), respectively. It consists of alternating Sb and As atoms in the atomic rows along the $a$ direction. This is the result of the short range anti-ordering vector $\underline{v}_{\mathrm{AO}}$ along the $a$ direction. Along the $m$ direction we chose the periodic continuation of these one-dimensional ordered rows. However, since the ordering in $m$ direction is weak, it is also conceivable that a certain fraction of the alternating $\mathrm{Sb}$ and As occupied atomic rows along the $a$ direction may be phase shifted (by one a lattice constant). Therefore, the ordering should be considered as a one-dimensional ordering in a two-dimensional single monolayer shell. This one-dimensional chemical order structure is named Iuliacumite, derived from the Latin name of the city of Jülich.

(a)

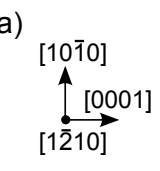

(b)
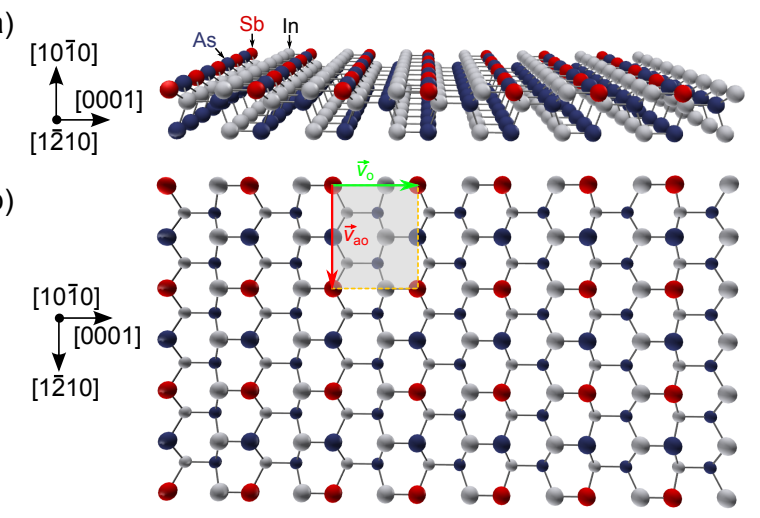

Figure 3: Perspective view of a ball model of the idealized, long-range, chemical ordering of the two-dimensional monolayer shell on the InAs sidewall facet, based on the ordering and anti-ordering vectors extracted from pair correlation function. (a) Side and (b) top view with surface unit cell (gray shaded area) and ordering as well as anti- ordering vectors shown as green and red arrows, respectively.

In conclusion, we identified a novel short-range chemical ordering type in single monolayer $\mathrm{InAs}_{1-x} \mathrm{Sb}_{x}$ wurtzite structure shells on $m$ plane sidewall facets of InAs nanowires by direct atomically resolved elemental mapping using scanning tunneling microscopy. The ordering is driven by strong $\mathrm{Sb}-\mathrm{Sb}$ repulsions along the atomic chains in a direction, giving rise to an anti-correlation ordering in non-polar directions, deviating from ordering in bulk WZ structure material observed thus far. This highlights the importance of spatial confinement for the formation of novel chemical ordering structures in III-V semiconductor alloys. Such chemical ordering effects in atomically thin structures may offer new degrees of adjusting 
and controlling the electronic properties in systems with reduced dimensions. Finally, it is also conceivable that the here observed ordering in a two-dimensional single monolayer may lead through exotic growth schemes to new types of bulk ordering.

The authors thank P. Caroff for his involvement in the nanowire growth and the European Community's H2020 program (Grant No. PITN-GA-2016-722176, Indeed Project), the EQUIPEX programs Excelsior (Grant No. ANR-11-EQPX-0015), the RENATECH network, the Deutsche Forschungsgemeinschaft (DFG, German research foundation) - 390247238, the Natural Science Foundation of Shanghai (19ZR1419500), and of the Region Nord-Pas-deCalais for financial support.

\section{References}

(1) Caroff, P.; Wagner, J. B.; Dick, K. A.; Nilsson, H. A.; Jeppsson, M.; Deppert, K.; Samuelson, L.; Wallenberg, L. R.; Wernersson, L. Small 2008, 4, 878-882.

(2) Xu, T.; Dick, K. A.; Plissard, S.; Nguyen, T. H.; Makoudi, Y.; Berthe, M.; Nys., J.-P.; Wallart, X.; Grandidier, B.; Caroff, P. Nanotechnol. 2012, 23, 095702.

(3) Gorji Ghalamestani, S.; Lehmann, S.; Dick, K. A. Nanoscale 2016, 8, 2778-2786.

(4) Wei, S.-H.; Zunger, A. Phys. Rev. B 1989, 39, 3279-3304.

(5) Zunger, A.; Mahajan, S. In Handbook on Semiconductors: Materials, Properties and Preparation; Mahajan, S., Ed.; North-Holland, 1994; Vol. 3B; pp 1399-1514.

(6) Gomyo, A.; Makita, K.; Hino, I.; Suzuki, T. Phys. Rev. Lett. 1994, 72, 673-676.

(7) Mascarenhas, A. Spontaneous Ordering in Semiconductor Alloys; Kluwer Academic / Plenum Publishers, New York, 2002.

(8) Wu, M.; Luna, E.; Puustinen, J.; Guina, M.; Trampert, A. Appl. Phys. Lett. 2014, 105, 041602. 
(9) Schnedler, M.; Lefebvre, I.; Xu, T.; Portz, V.; Patriarche, G.; Nys, J.-P.; Plissard, S. R.; Caroff, P.; Berthe, M.; Eisele, H.; Dunin-Borkowski, R. E.; Ebert, P.; Grandidier, B. Phys. Rev. B 2016, 94, 195306.

(10) Albrecht, M.; Lymperakis, L.; Neugebauer, J.; Northrup, J. E.; Kirste, L.; Leroux, M.; Grzegory, I.; Porowski, S.; Strunk, H. P. Phys. Rev. B 2005, 71, 035314.

(11) Korakakis, D.; Ludwig, K. F.; Moustakas, T. D. Appl. Phys. Lett. 1997, 71, 72-74.

(12) Iliopoulos, E.; Ludwig, K. F.; Moustakas, T. D. J. Phys. Chem. Solids 2003, 64, 1525 $-1532$.

(13) Woicik, J. C.; Ludwig, K. F.; Moustakas, T. D. Appl. Phys. Lett. 2012, 100, 162105.

(14) Gao, M.; Lin, Y.; Bradley, S. T.; Ringel, S. A.; Hwang, J.; Schaff, W. J.; Brillson, L. J. Appl. Phys. Lett. 2005, 87, 191906.

(15) Wu, Z. H.; Kawai, Y.; Fang, Y.-Y.; Chen, C. Q.; Kondo, H.; Hori, M.; Honda, Y.; Yamaguchi, M.; Amano, H. Appl. Phys. Lett. 2011, 98, 141905.

(16) Woo, S. Y.; Bugnet, M.; Nguyen, H. P. T.; Mi, Z.; Botton, G. A. Nano Lett. 2015, 15, 6413-6418.

(17) Ruterana, P.; De Saint Jores, G.; Laügt, M.; Omnes, F.; Bellet-Amalric, E. Appl. Phys. Lett. 2001, 78, 344-346.

(18) Benamara, M.; Kirste, L.; Albrecht, M.; Benz, K. W.; Strunk, H. Appl. Phys. Lett. 2003, 82, 547-549.

(19) Laaksonen, K.; Ganchenkova, M.; Nieminen, R. Physica B 2006, 376-377, 502 - 506.

(20) Pavloudis, T.; Kioseoglou, J.; Karakostas, T.; Komninou, P. Comp. Mat. Sci. 2016, $118,22-31$.

(21) Komsa, H.-P.; Krasheninnikov, A. V. J. Phys. Chem. Lett. 2012, 3, $3652-3656$. 
(22) Chen, Y.; Xi, J.; Dumcenco, D. O.; Liu, Z.; Suenaga, K.; Wang, D.; Shuai, Z.; Huang, Y.-S.; Xie, L. ACS Nano 2013, 7, 4610-4616.

(23) Tongay, S.; Narang, D. S.; Kang, J.; Fan, W.; Ko, C.; Luce, A. V.; Wang, K. X.; Suh, J.; Patel, K. D.; Pathak, V. M.; Li, J.; Wu, J. Appl. Phys. Lett. 2014, 104, 012101.

(24) Siemens, B.; Domke, C.; Ph. Ebert,; Urban, K. Phys. Rev. B 1997, 56, 12321-12326.

(25) Steinshnider, J.; Harper, J.; Weimer, M.; Lin, C.-H.; Pei, S. S.; Chow, D. H. Phys. Rev. Lett. 2000, 85, 4562-4565.

(26) Pfister, M.; Johnson, M. B.; Alvarado, S. F.; Salemink, H. W. M.; Marti, U.; Martin, D.; Morier-Genoud, F.; Reinhart, F. K. Appl. Phys. Lett. 1995, 67, 1459-1461.

(27) Ph. Ebert,; Heinrich, M.; Simon, M.; Domke, C.; Urban, K.; Shih, C.; Webb, M.; Lagally, M. Phys. Rev. B 1996, 53, 4580-4590.

(28) Chao, K.-J.; Shih, C.-K.; Gotthold, D. W.; Streetman, B. G. Phys. Rev. Lett. 1997, $79,4822-4825$.

(29) McKay, H. A.; Feenstra, R. M.; Schmidtling, T.; Pohl, U. W. Appl. Phys. Lett. 2001, $78,82-84$.

(30) Hjort, M.; Kratzer, P.; Lehmann, S.; Patel, S. J.; Dick, K. A.; Palmstrøm, C. J.; Timm, R.; Mikkelsen, A. Nano Lett. 2017, 17, 3634-3640.

(31) Borg, B. M.; Wernersson, L.-E. Nanotechnol. 2013, 24, 202001.

(32) The mean force potential equals the pair interaction energy in the low density limit under equilibrium. T. L. Hill, Statistical Mechanics (McGraw-Hill, New York, 1956).

(33) Kresse, G.; Furthmüller, J. Phys. Rev. B 1996, 54, 11169-11186.

(34) Blöchl, P. E. Phys. Rev. B 1994, 50, 17953-17979. 
(35) Ceperley, D. M.; Alder, B. J. Phys. Rev. Lett. 1980, 45, 566-569.

(36) Ph. Ebert,; Zhang, T.; Kluge, F.; Simon, M.; Zhang, Z.; Urban, K. Phys. Rev. Lett. 1999, 83, 757-760. 